\title{
A Detailed Normal-Mode Energetics of the General Circulation of the Atmosphere
}

\author{
C. A. F. Marques and J. M. Castanheira \\ CESAM, and Department of Physics, University of Aveiro, Aveiro, Portugal
}

(Manuscript received 24 November 2011, in final form 26 March 2012)

\begin{abstract}
An energetics formulation is here introduced that enables an explicit evaluation for the conversion rates between available potential energy and kinetic energy, the nonlinear interactions of both energy forms, and their generation and dissipation rates, in both the zonal wavenumber and vertical mode domains. The conversion rates between available potential energy and kinetic energy are further decomposed into the contributions by the rotational (Rossby) and divergent (gravity) components of the circulation field. The computed energy terms allow one to formulate a detailed energy cycle describing the flow of energy among the zonal mean and eddy components, and also among the barotropic and baroclinic components. This new energetics formulation is a development of the $3 \mathrm{D}$ normal-mode energetics scheme. The new formulation is applied on an assessment of the energetics of winter (December-February) circulation in the 40-yr ECMWF Re-Analysis (ERA-40), the 25-yr Japan Meteorological Agency Reanalysis (JRA-25), and the NCEPDepartment of Energy Reanalysis 2 (NCEP-R2) datasets.
\end{abstract}

\section{Introduction}

Since the global energy cycle in the atmosphere was introduced by Lorenz (1955), the energetics of the atmospheric general circulation has been further investigated with orthogonal projections of the circulation field onto various basis functions. Saltzman (1957) presented the energetics in the zonal wavenumber domain, using a zonal harmonic expansion, which allows for the analysis of energy amounts and energy conversion/transfer rates in eddies of given wavenumber as well as the interaction between the eddies. Kao (1968) and Hayashi (1980) extended the approach of Saltzman (1957) to the wavenumber-frequency domain using a two-dimensional Fourier expansion. Other expansions were also pursued in the zonal and meridional directions using a spherical harmonic expansion (Eliasen and Machenhauer 1965; Lambert 1984), and in the vertical direction with empirical orthogonal functions (Holmström 1963). Tanaka (1985) and Tanaka and Kung (1988) developed a threedimensional (3D) normal-mode energetics scheme, using the $3 \mathrm{D}$ normal modes of a set of linearized primitive equations as a basis to expand the global circulation field.

\footnotetext{
Corresponding author address: Carlos Marques, Departamento de Física da Universidade de Aveiro, Campus Universitário de Santiago, 3810-193 Aveiro, Portugal.

E-mail: cafm@ua.pt
}

The 3D normal-mode energetics (NME) combines three one-dimensional spectral energetics in domains of zonal wavenumber $n$, a meridional mode number $l$, and a vertical mode number $k$. The scheme complement the standard energetics in the zonal wavenumber domain, since it can diagnose the 3D spectral distribution of energy and energy interactions, the energetics characteristics of Rossby waves and gravity waves, and the energy interaction between the barotropic and baroclinic modes (Tanaka and Kung 1988). That is, by summing up the 3D NME terms within the same physical categories, the energetics characteristics can be assessed separately, not only for the zonal mean and eddy components, but also for the barotropic and baroclinic modes, and for the Rossby and gravity waves.

A drawback of the 3D NME is that only the total energy, that is, the kinetic plus the available potential energies $E_{n l k}$ associated with each mode can be calculated, and a separate analysis of the available potential and kinetic energies, as well as of their conversions, cannot be performed in the 3D framework. However, as we will show here, that separation can be performed in both the vertical and wavenumber domains. In fact, the energetics formulation presented in this study performs an explicit evaluation of the available potential energy and kinetic energy as well as the conversion rates between them, along with their generation and dissipation rates and the nonlinear interactions of each energy form, 
in both the zonal wavenumber and vertical mode domains. The calculated energetics terms are used to construct a detailed energy cycle diagram that describes the flow of energy in the atmosphere decomposed into the zonal mean and eddy components, and also into the barotropic and baroclinic components.

The energetics scheme here developed is applied to an assessment of the energetics in three reanalysis datasets, namely the National Centers for Environmental Prediction (NCEP) and Department of Energy (DOE) second Atmospheric Model Intercomparison Project (AMIP-II) Reanalysis (hereafter called NCEP-R2); the 40-yr European Centre for Medium-Range Weather Forecasts (ECMWF) Re-Analysis (ERA-40); and the 25-yr reanalysis produced by the Japan Meteorological Agency (JMA) and the Central Research Institute of Electric Power Industry (CRIEPI) (JRA-25).

\section{Analysis scheme}

The equations of horizontal motion and thermodynamics with three dependent variables $(u, v, \phi)$ may be written in the $(\lambda, \theta, p, t)$ coordinate system as

$$
\frac{\partial u}{\partial t}-f v+\frac{1}{a \cos \theta} \frac{\partial \phi}{\partial \lambda}=-\mathbf{V} \cdot \nabla u-\omega \frac{\partial u}{\partial p}+\frac{\tan \theta}{a} u v+F_{u}
$$

$$
\begin{aligned}
& \frac{\partial v}{\partial t}+f u+\frac{1}{a} \frac{\partial \phi}{\partial \theta}=-\mathbf{V} \cdot \nabla v-\omega \frac{\partial v}{\partial p}-\frac{\tan \theta}{a} u^{2}+F_{v}, \\
& -\frac{\partial}{\partial t}\left[\frac{\partial}{\partial p}\left(\frac{1}{S_{0}} \frac{\partial \phi}{\partial p}\right)\right]+\nabla \cdot \mathbf{V}=\frac{\partial}{\partial p}\left[\frac{R}{p S_{0}}\left(-\mathbf{V} \cdot \nabla T-\omega \frac{\partial T}{\partial p}\right)\right] \\
& +\frac{\partial}{\partial p}\left(\frac{q R}{p c_{p} S_{0}}\right)
\end{aligned}
$$

where $T$ and $\phi$ are the departures of the temperature and geopotential from the reference state $T_{0}(p)$ and $\phi_{0}(p)$, respectively, and $S_{0}$ is the static stability parameter of the reference state:

$$
S_{0}=\frac{R}{p}\left(\frac{R T_{0}}{p c_{p}}-\frac{d T_{0}}{d p}\right)
$$

The variable $q$ is the rate of diabatic heating per mass unity, and the remaining symbols are mostly customary. Since a scaling of $T_{0} \gg T$ has been introduced in Eq. (3), the associated term $\omega R T / p c_{p}$ has been neglected.

The right-hand sides of Eqs. (1)-(3) contain the nonlinear terms, frictional forces, and the diabatic heat sources. Considering an inviscid and adiabatic atmosphere, the linearized versions of Eqs. (1)-(3) about a reference state at rest with a mean static stability parameter $S_{0}$ is obtained by setting their right-hand side to zero. The free oscillations - normal modes - of this linearized system may be written in the form

$$
\left(\begin{array}{l}
u \\
v \\
\phi
\end{array}\right)_{n l k}=e^{-i 2 \Omega \sigma t} G_{k}(p) e^{i n \lambda} \mathbf{X}_{k} \cdot\left[\begin{array}{c}
U(\theta) \\
i V(\theta) \\
\Phi(\theta)
\end{array}\right]_{n l k},
$$

where $\mathbf{X}_{k}=\operatorname{diag}\left(\sqrt{g h_{k}}, \sqrt{g h_{k}}, g h_{k}\right)$ is a diagonal matrix of scaling factors, $g$ being the earth's gravity, $\sigma$ a dimensionless frequency, and $h_{k}$ the equivalent height. Also, $G_{k}(p)$ is a separable vertical structure function and $k$ is the respective vertical index. Because $G_{0}(p)$ has no node and is approximately constant, the modes for $k=0$ are called external or barotropic. The modes for $k \geq 1$ have $k$ nodes and are called internal or baroclinic. Each horizontal structure function is given by the product of a zonal wave with wavenumber $n$ and a vector $[U(\theta), i V(\theta), \Phi(\theta)]_{n l k}^{\mathrm{T}}$ that defines the meridional profile of the wave. Because the meridional index $l$ is associated with the number of zeros of the respective meridional profile, it may be regarded as an index of the meridional scale of the motion and is defined as a sequence of three distinct modes, $l_{r}, l_{w}$, and $l_{e}$, in order to distinguish each wave type (i.e., the westward-propagating Rossby wave and the westward- and eastward-propagating gravity waves, respectively).

The normal modes form a complete orthogonal basis and therefore allow expanding the horizontal wind and the geopotential fields of the global atmosphere (e.g., Daley 1991; Cohn and Dee 1989; Tanaka 1985):

$$
\left(\begin{array}{l}
u \\
v \\
\phi
\end{array}\right)=\sum_{n=-\infty}^{\infty} \sum_{l=0}^{\infty} \sum_{k=0}^{\infty} w_{n l k}(t) G_{k}(p) e^{i n \lambda} \mathbf{X}_{k} \cdot\left[\begin{array}{c}
U(\theta) \\
i V(\theta) \\
\Phi(\theta)
\end{array}\right]_{n l k}
$$

The expansion coefficients $w_{n l k}$ are obtained by means of a vertical projection onto the vertical structure functions,

$$
(u, v, \phi)_{k}^{\mathrm{T}}=\frac{1}{p_{s}} \int_{0}^{p_{s}}(u, v, \phi)^{\mathrm{T}} G_{k}(p) d p,
$$

followed by an horizontal projection onto the horizontal structure functions:

$$
w_{n l k}=\frac{1}{2 \pi} \int_{0}^{2 \pi} \int_{-\pi / 2}^{\pi / 2} \mathbf{H}_{n l k}^{*} \mathbf{X}_{k}^{-1} \cdot(u, v, \phi)_{k}^{\mathrm{T}} \cos \theta d \theta d \lambda .
$$

We have assumed that the vertical structure functions $G_{k}(p)$ and the horizontal structure functions $\mathbf{H}_{n l k}(\lambda, \theta)=$ $e^{i n \lambda}[U(\theta), i V(\theta), \Phi(\theta)]_{n l k}^{\mathrm{T}}$ have unitary norms. The surface 
pressure $p_{s}$ is treated as a constant and the superscript T and the asterisk respectively denote the transpose and the complex conjugate of the transpose.

\section{Energy balance equations for the normal modes}

Multiplying the linearized versions of Eqs. (1), (2), and (3) by $u, v$, and $\phi$, respectively, summing the three resulting equations, integrating over the whole atmospheric mass, and using the upper boundary condition

$$
\lim _{p \rightarrow 0}(\phi \omega)=0
$$

which would guarantee energy conservation (Cohn and Dee 1989), the equation of energy conservation is obtained as

$$
\frac{d}{d t}\left[\frac{1}{g S} \int_{S}\left(\int_{0}^{p_{s}} E d p+\frac{1}{2} \frac{p_{s}}{R T_{s}} \phi_{s}^{2}\right) d S\right]=0
$$

where

$$
\begin{aligned}
& E=K+A, \\
& K=\frac{1}{2}\left(u^{2}+v^{2}\right), \\
& A=\frac{1}{2 S_{0}}\left(\frac{\partial \phi}{\partial p}\right)^{2},
\end{aligned}
$$

and the subscript $s$ denotes the variables at $p=p_{s}$. The total energy in the normal mode energetics includes contributions from the gravitational potential energy due to vertical displacement of the lower boundary, which is expressed by the surface integral in Eq. (10). By expanding the dependent variables onto the vertical normal modes using Eq. (7), Eq. (10) takes the form

$$
\frac{d}{d t}\left[\sum_{k=0}^{\infty} \frac{p_{s} h_{k}}{2 S} \int_{S}\left(\hat{u}_{k}^{2}+\hat{v}_{k}^{2}+\hat{\phi}_{k}^{2}\right) d S\right]=0,
$$

where dimensionless variables were considered:

$$
\hat{u}_{k}=\frac{u_{k}}{\sqrt{g h_{k}}}, \quad \hat{v}_{k}=\frac{v_{k}}{\sqrt{g h_{k}}}, \quad \hat{\phi}_{k}=\frac{\phi_{k}}{g h_{k}} .
$$

Expanding Eq. (14) onto the Fourier-Hough harmonics using Eq. (8), the energy conservation is finally expressed in terms of a summation of energies associated with each mode; that is,

$$
\frac{d}{d t} \sum_{n=0}^{\infty} \sum_{l=0}^{\infty} \sum_{k=0}^{\infty} E_{n l k}=0
$$

where

$$
\begin{aligned}
& E_{0 l k}=\frac{1}{4} p_{s} h_{k}\left|w_{0 l k}\right|^{2}, \\
& E_{n l k}=\frac{1}{2} p_{s} h_{k}\left|w_{n l k}\right|^{2} .
\end{aligned}
$$

Tanaka (1985) and Tanaka and Kung (1988) used the full projection of the complete nonlinear equations (1)(3) onto the 3D normal modes to devise an energetics scheme that allows the spectral analysis of the total energy and energy interactions in the domains of the zonal wavenumber, the meridional mode number, and the vertical mode number, as well as its partition between Rossby and gravity modes or barotropic and baroclinic modes. As already mentioned, the energetics scheme developed by Tanaka (1985) only computes the total energy and does not allow for a separate assessment of the kinetic and available potential energies or for the conversions between the two types of energy.

As we will show below, if only a partial projection of the complete nonlinear equations (1)-(3) onto the vertical and zonal components is performed, it is possible to analyze separately the kinetic and available potential energies as well as the conversions between the two forms of energy. Furthermore, if the $(u, v, \phi)$ fields are partitioned into Rossby and gravity by retaining only Rossby or gravity normal modes in the expansion in Eq. (6), we may assess the role of the Rossby and gravity waves in the conversions between the two forms of energy.

Applying the vertical projection in Eq. (7) to the horizontal momentum equations (1) and (2), one obtains

$$
\begin{gathered}
\frac{\partial \hat{u}_{k}}{\partial \hat{t}}-\sin \theta \hat{v}_{k}+\frac{\alpha_{k}}{\cos \theta} \frac{\partial \hat{\phi}_{k}}{\partial \lambda}=\left(\hat{I}_{1}\right)_{k}+\left(\hat{F}_{u}\right)_{k} \\
\frac{\partial \hat{v}_{k}}{\partial \hat{t}}+\sin \theta \hat{u}_{k}+\alpha_{k} \frac{\partial \hat{\phi}_{k}}{\partial \theta}=\left(\hat{I}_{2}\right)_{k}+\left(\hat{F}_{v}\right)_{k}
\end{gathered}
$$

where

$$
\hat{t}=2 \Omega t,
$$

$$
\begin{aligned}
& \left(\hat{F}_{u}\right)_{k}=\frac{\left(F_{u}\right)_{k}}{2 \Omega \sqrt{g h_{k}}}, \quad\left(\hat{F}_{v}\right)_{k}=\frac{\left(F_{v}\right)_{k}}{2 \Omega \sqrt{g h_{k}}}, \\
& \left(\hat{I}_{1}\right)_{k}=\frac{1}{2 \Omega \sqrt{g h_{k}}}\left(-\mathbf{V} \cdot \nabla u-\omega \frac{\partial u}{\partial p}+\frac{\tan \theta}{a} u v\right)_{k}, \\
& \left(\hat{I}_{2}\right)_{k}=\frac{1}{2 \Omega \sqrt{g h_{k}}}\left(-\mathbf{V} \cdot \nabla v-\omega \frac{\partial v}{\partial p}-\frac{\tan \theta}{a} u^{2}\right)_{k} .
\end{aligned}
$$

The constant $\Omega$ is the angular frequency of the earth's rotation and the dimensionless coefficient $\alpha_{k}$ is defined as 


$$
\alpha_{k}=\frac{\sqrt{g h_{k}}}{2 \Omega a} .
$$

Applying the Fourier transform to Eqs. (19) and (20) yields

$$
\frac{\partial \hat{u}_{n k}}{\partial \hat{t}}=\sin \theta \hat{v}_{n k}-\frac{i n \alpha_{k}}{\cos \theta} \hat{\phi}_{n k}+\left(\hat{I}_{1}\right)_{n k}+\left(\hat{F}_{u}\right)_{n k},
$$

$\frac{\partial \hat{v}_{n k}}{\partial \hat{t}}=-\sin \theta \hat{u}_{n k}-\alpha_{k} \frac{\partial \hat{\phi}_{n k}}{\partial \theta}+\left(\hat{I}_{2}\right)_{n k}+\left(\hat{F}_{v}\right)_{n k}$.

Multiplying Eqs. (26) and (27) by $\hat{u}_{n k}^{*}$ and $\hat{v}_{n k}^{*}$, respectively, and summing each resulting equation with the corresponding complex conjugate equation gives

$$
\begin{aligned}
\frac{\partial}{\partial \hat{t}}\left(\hat{u}_{n k}^{*} \hat{u}_{n k}\right)= & \sin \theta\left(\hat{u}_{n k}^{*} \hat{v}_{n k}+\hat{u}_{n k} \hat{v}_{n k}^{*}\right)+\frac{i n \alpha_{k}}{\cos \theta}\left(\hat{u}_{n k} \hat{\phi}_{n k}^{*}-\hat{u}_{n k}^{*} \hat{\phi}_{n k}\right)+\left[\hat{u}_{n k}^{*}\left(\hat{I}_{1}\right)_{n k}\right. \\
& \left.+\hat{u}_{n k}\left(\hat{I}_{1}\right)_{n k}^{*}\right]+\left[\hat{u}_{n k}^{*}\left(\hat{F}_{u}\right)_{n k}+\hat{u}_{n k}\left(\hat{F}_{u}\right)_{n k}^{*}\right], \\
\frac{\partial}{\partial \hat{t}}\left(\hat{v}_{n k}^{*} \hat{v}_{n k}\right)= & -\sin \theta\left(\hat{v}_{n k}^{*} \hat{u}_{n k}+\hat{v}_{n k} \hat{u}_{n k}^{*}\right)-\alpha_{k}\left(\hat{v}_{n k}^{*} \frac{\partial \hat{\phi}_{n k}}{\partial \theta}+\hat{v}_{n k} \frac{\partial \hat{\phi}_{n k}^{*}}{\partial \theta}\right) \\
& +\left[\hat{v}_{n k}^{*}\left(\hat{I}_{2}\right)_{n k}+\hat{v}_{n k}\left(\hat{I}_{2}\right)_{n k}^{*}\right]+\left[\hat{v}_{n k}^{*}\left(\hat{F}_{v}\right)_{n k}+\hat{v}_{n k}\left(\hat{F}_{v}\right)_{n k}^{*}\right] .
\end{aligned}
$$

Finally, summing Eqs. (28) and (29), multiplying the resulting equation by $p_{s} h_{k} / 2$, integrating over the latitude, and recalling that $\hat{t}=2 \Omega t$, the rate of change of kinetic energy in the zonal wavenumber and vertical mode number domains may be written as

$$
\frac{\partial}{\partial t} K_{n k}=C_{n k}+I_{n k}-D_{n k}
$$

where

$$
\begin{aligned}
K_{n k} & =\frac{p_{s} h_{k}}{2} \int_{-\pi / 2}^{\pi / 2}\left(\hat{u}_{n k}^{*} \hat{u}_{n k}+\hat{v}_{n k}^{*} \hat{v}_{n k}\right) \cos \theta d \theta, \\
C_{n k} & =p_{s} \Omega h_{k} \int_{-\pi / 2}^{\pi / 2}\left[\frac{i n \alpha_{k}}{\cos \theta}\left(\hat{u}_{n k} \hat{\phi}_{n k}^{*}-\hat{u}_{n k}^{*} \hat{\phi}_{n k}\right)-\alpha_{k}\left(\hat{v}_{n k}^{*} \frac{\partial \hat{\phi}_{n k}}{\partial \theta}+\hat{v}_{n k} \frac{\partial \hat{\phi}_{n k}^{*}}{\partial \theta}\right)\right] \cos \theta d \theta, \\
I_{n k} & =p_{s} \Omega h_{k} \int_{-\pi / 2}^{\pi / 2}\left\{\left[\hat{u}_{n k}^{*}\left(\hat{I}_{1}\right)_{n k}+\hat{u}_{n k}\left(\hat{I}_{1}\right)_{n k}\right]+\left[\hat{v}_{n k}^{*}\left(\hat{I}_{2}\right)_{n k}+\hat{v}_{n k}\left(\hat{I}_{2}\right)_{n k}^{*}\right]\right\} \cos \theta d \theta, \\
D_{n k} & =-p_{s} \Omega h_{k} \int_{-\pi / 2}^{\pi / 2}\left\{\left[\hat{u}_{n k}^{*}\left(\hat{F}_{u}\right)_{n k}+\hat{u}_{n k}\left(\hat{F}_{u}\right)_{n k}^{*}\right]+\left[\hat{v}_{n k}^{*}\left(\hat{F}_{v}\right)_{n k}+\hat{v}_{n k}\left(\hat{F}_{v}\right)_{n k}^{*}\right]\right\} \cos \theta d \theta .
\end{aligned}
$$

Following a similar procedure for the thermodynamic energy equation (3), the rate of change of available potential energy in the zonal wavenumber and vertical mode number domains may be written as

$$
\frac{\partial}{\partial t} A_{n k}=-C_{n k}+J_{n k}+G_{n k}
$$

where

$$
A_{n k}=\frac{p_{s} h_{k}}{2} \int_{-\pi / 2}^{\pi / 2} \hat{\phi}_{n k}^{*} \hat{\phi}_{n k} \cos \theta d \theta
$$

$$
J_{n k}=p_{s} \Omega h_{k} \int_{-\pi / 2}^{\pi / 2}\left[\hat{\phi}_{n k}^{*}\left(\hat{J}_{3}\right)_{n k}+\hat{\phi}_{n k}\left(\hat{J}_{3}\right)_{n k}^{*}\right] \cos \theta d \theta,
$$

$$
G_{n k}=p_{s} \Omega h_{k} \int_{-\pi / 2}^{\pi / 2}\left[\hat{\phi}_{n k}^{*}\left(\hat{N}_{3}\right)_{n k}+\hat{\phi}_{n k}\left(\hat{N}_{3}\right)_{n k}^{*}\right] \cos \theta d \theta,
$$

and

$$
\hat{J}_{3}=\frac{1}{2 \Omega} \frac{\partial}{\partial p}\left[\frac{R}{p S_{0}}\left(-\mathbf{V} \cdot \nabla T-\omega \frac{\partial T}{\partial p}\right)\right]
$$




$$
\hat{N}_{3}=\frac{1}{2 \Omega}\left[\frac{\partial}{\partial p}\left(\frac{q R}{p c_{p} S_{0}}\right)\right] \text {. }
$$

The term $C_{n k}$ appears in both Eqs. (30) and (35) with opposite signs, and therefore represents the conversion rate between available potential energy and kinetic energy in both the zonal wavenumber and vertical mode domains. The nonlinear interactions of kinetic and available potential energies in the zonal wavenumber and vertical mode domains are represented by the terms $I_{n k}$ and $J_{n k}$, respectively. On the other hand, the terms $D_{n k}$ and $G_{n k}$ represent the dissipation of kinetic energy and the generation of available potential energy due to diabatic processes.

Taking the full projection of $(u, v, \phi)$ onto the 3D normal modes, the vertical and zonal transforms may be obtained separately for Rossby and gravity fields using the following expansions:

$$
\begin{aligned}
& \left(\begin{array}{c}
\hat{u}_{n k}^{R} \\
\hat{v}_{n k}^{R} \\
\hat{\phi}_{n k}^{R}
\end{array}\right)=\sum_{l_{r}=0}^{L_{R}} w_{n l_{r} k} \boldsymbol{\Theta}_{n l_{r} k}(\theta), \\
& \left(\begin{array}{c}
\hat{u}_{n k}^{G} \\
\hat{v}_{n k}^{G} \\
\hat{\phi}_{n k}^{G}
\end{array}\right)=\sum_{l_{w}=0}^{L_{W}} w_{n l_{w} k} \boldsymbol{\Theta}_{n l_{w} k}(\theta)+\sum_{l_{e}=0}^{L_{E}} w_{n l_{e} k} \boldsymbol{\Theta}_{n l_{e} k}(\theta),
\end{aligned}
$$

where $L_{R}, L_{W}$, and $L_{E}$ are the total number of meridional modes of each type (i.e., Rossby, westward gravity, and eastward gravity, respectively) used for the computation of the Hough vector functions $\boldsymbol{\Theta}_{n l k}(\theta)$. Following this procedure, the conversion rates may be decomposed as follows:

$$
C_{n k}=C_{n k}^{R R}+C_{n k}^{R G}+C_{n k}^{G R}+C_{n k}^{G G},
$$

where

$$
\begin{aligned}
& C_{n k}^{R R}=p_{s} \Omega h_{k} \int_{-\pi / 2}^{\pi / 2}\left[\frac{i n \alpha_{k}}{\cos \theta}\left(\hat{u}_{n k}^{R} \hat{\phi}_{n k}^{* R}-\hat{u}_{n k}^{* R} \hat{\phi}_{n k}^{R}\right)-\alpha_{k}\left(\hat{v}_{n k}^{* R} \frac{\partial \hat{\phi}_{n k}^{R}}{\partial \theta}+\hat{v}_{n k}^{R} \frac{\partial \hat{\phi}_{n k}^{* R}}{\partial \theta}\right)\right] \cos \theta d \theta, \\
& C_{n k}^{R G}=p_{s} \Omega h_{k} \int_{-\pi / 2}^{\pi / 2}\left[\frac{i n \alpha_{k}}{\cos \theta}\left(\hat{u}_{n k}^{R} \hat{\phi}_{n k}^{* G}-\hat{u}_{n k}^{* R} \hat{\phi}_{n k}^{G}\right)-\alpha_{k}\left(\hat{v}_{n k}^{*} \frac{\partial \hat{\phi}_{n k}^{G}}{\partial \theta}+\hat{v}_{n k}^{R} \frac{\partial \hat{\phi}_{n k}^{*}}{\partial \theta}\right)\right] \cos \theta d \theta, \\
& C_{n k}^{G R}=p_{s} \Omega h_{k} \int_{-\pi / 2}^{\pi / 2}\left[\frac{i n \alpha_{k}}{\cos \theta}\left(\hat{u}_{n k}^{G} \hat{\phi}_{n k}^{* R}-\hat{u}_{n k}^{* G} \hat{\phi}_{n k}^{R}\right)-\alpha_{k}\left(\hat{v}_{n k}^{* G} \frac{\partial \hat{\phi}_{n k}^{R}}{\partial \theta}+\hat{v}_{n k}^{G} \frac{\partial \hat{\phi}_{n k}^{*}}{\partial \theta}\right)\right] \cos \theta d \theta, \\
& C_{n k}^{G G}=p_{s} \Omega h_{k} \int_{-\pi / 2}^{\pi / 2}\left[\frac{i n \alpha_{k}}{\cos \theta}\left(\hat{u}_{n k}^{G} \hat{\phi}_{n k}^{* G}-\hat{u}_{n k}^{* G} \hat{\phi}_{n k}^{G}\right)-\alpha_{k}\left(\hat{v}_{n k}^{*} \frac{\partial \hat{\phi}_{n k}^{G}}{\partial \theta}+\hat{v}_{n k}^{G} \frac{\partial \hat{\phi}_{n k}^{* G}}{\partial \theta}\right)\right] \cos \theta d \theta .
\end{aligned}
$$

The terms $C_{n k}^{R R}$ and $C_{n k}^{G G}$ represent the conversion rates of available potential energy into kinetic energy due to the Rossby and gravity waves, respectively. The term $C_{n k}^{R G}$ is the conversion rate due to the work of the pressure gradient force of the divergent (gravity) adjusted mass field on the rotational (Rossby) motion, whereas $C_{n k}^{G R}$ represents the energy conversion due to the work of the pressure gradient force of the rotational adjusted mass field on the divergent motion.

\section{Data and methods}

The variables used in the energetics computation are temperature, the three wind components, and the geopotential height. For NCEP-R2, 6-hourly values of these variables at 17 pressure levels $(1000,925,850,700,600,500$, $400,300,250,200,150,100,70,50,30,20$, and $10 \mathrm{hPa}$ ) with a horizontal resolution of $2.5^{\circ}$ longitude $\times 2.5^{\circ}$ latitude are used. The same resolutions were considered for the other two datasets, except for ERA-40, for which 23 pressure levels (the same as NCEP-R2 plus 750, 7, 5, 3, 2, and $1 \mathrm{hPa}$ ) are used, and JRA-25 for which 23 pressure levels (the same as NCEP-R2 plus 7, 5, 3, 2,1, and $0.4 \mathrm{hPa}$ ) are used. The period 1979-2001 is selected for the analysis.

The vertical structure functions were computed using a reference state of the global mean temperature averaged over the periods considered for each dataset. The vertical structure equation (VSE) was solved by a spectral method as in Castanheira et al. (1999), which has the advantage, over the finite-difference method, that the derivatives of the vertical structure function can be calculated by analytical differentiation. In this study, 23 Legendre polynomials were used to approximate the solution of VSE and 15 vertical modes were retained. The Hough vector 

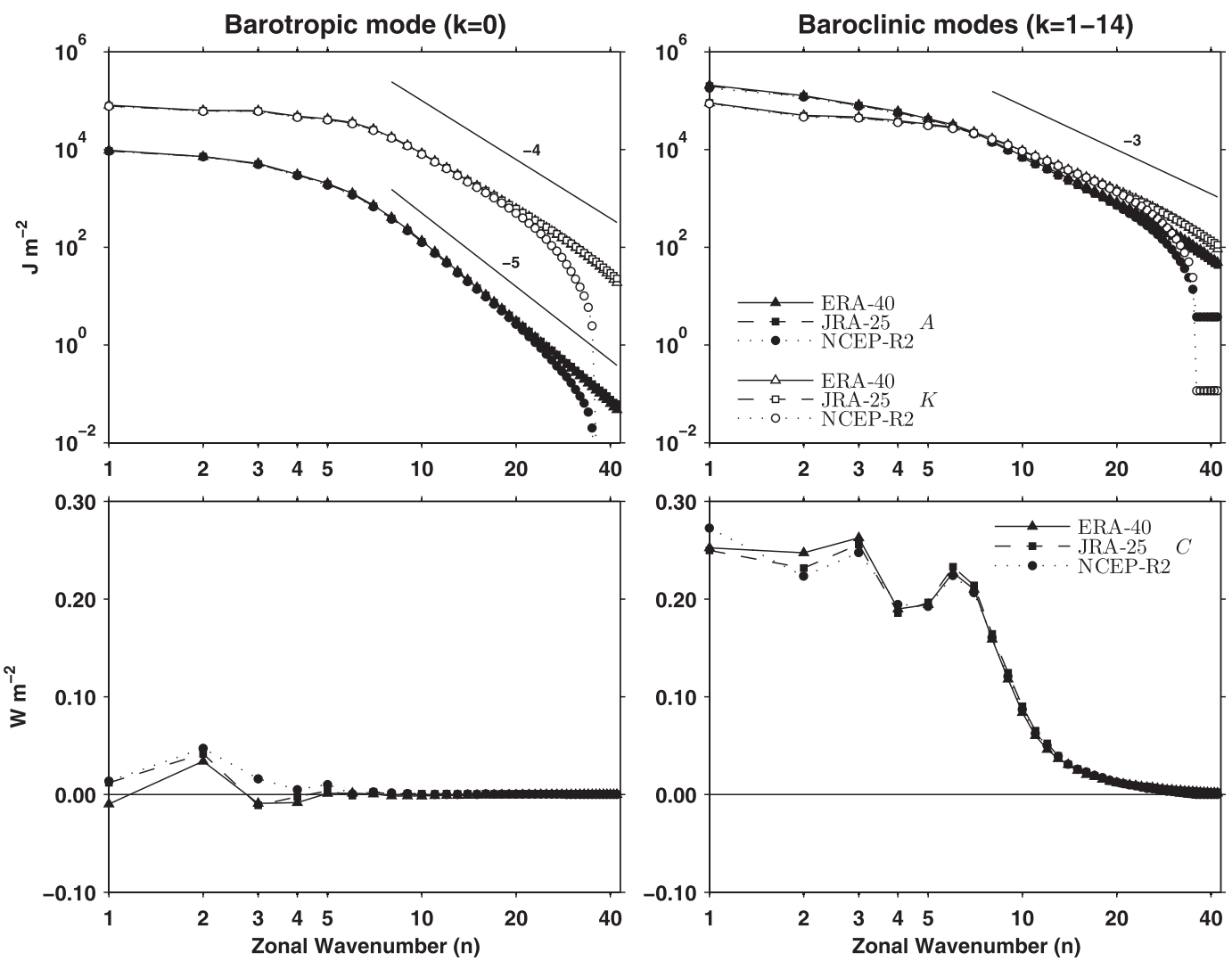

FIG. 1. Zonal wavenumber spectra of (top) available potential and kinetic energies ( $\left.\mathrm{J} \mathrm{m}^{-2}\right), A$ (black symbols) and $K$ (white symbols), and of (bottom) the conversion rate of available potential energy into kinetic energy $C\left(\mathrm{~W} \mathrm{~m}^{-2}\right)$ for ERA-40, JRA-25, and NCEP-R2 in DJF. The spectra are presented separately for the (left) barotropic and (right) baroclinic modes.

functions were computed following the method described in the paper of Swarztrauber and Kasahara (1985). The zonal wavenumber has been truncated at $n=42$. The modes for $n=0$ were determined following the approach suggested by Shigehisa (1983), which is also described in Swarztrauber and Kasahara (1985). In the following analysis only 40 Rossby modes and 20 eastward and 20 westward gravity modes were retained.

Finally, the energetics terms are computed following the analysis scheme represented by balance equations (30) and (35), where the generation and dissipation terms are computed as residuals. Equation (43) is also used to compute the conversion rates between the two forms of energy decomposed into the contributions by the rotational and divergent components of the circulation field. The energetics terms were computed at each time step (6-hourly) for all datasets and then averaged over the selected periods to obtain the corresponding mean values in the northern winter season [December-February (DJF)].

The assumption of vanishing winds at the lower boundary of the atmosphere has been adopted as in Tanaka and Kung (1988), in order to obtain a set of equations satisfying the energy conservation principle. As discussed in Tanaka and Kung (1988) and Tanaka (1994), the vanishing surface wind seems a reasonable and physically correct assumption for the normal-mode energetics. This nonslip condition has a very small effect in all energetics terms, except in the barotropic component of nonlinear interactions of available potential energy. Vanishing winds at the surface imply vanishing temperature advection there, and this would be the required condition to have energy conservation in an adiabatic atmosphere, bounded by an adiabatic surface at $p_{s}$.

\section{Results and discussion}

A $\log -\log$ scale is used to display the energy spectra in the wavenumber domain in order to assess their power-law behavior, whereas for the energy interactions and transformations a semilog scale is used in the wavenumber domain.

\section{a. Energetics in the zonal wavenumber and vertical mode domains}

Figures 1 and 2 show (top) the spectra of available potential and kinetic energies, $A$ (black symbols) and $K$ 

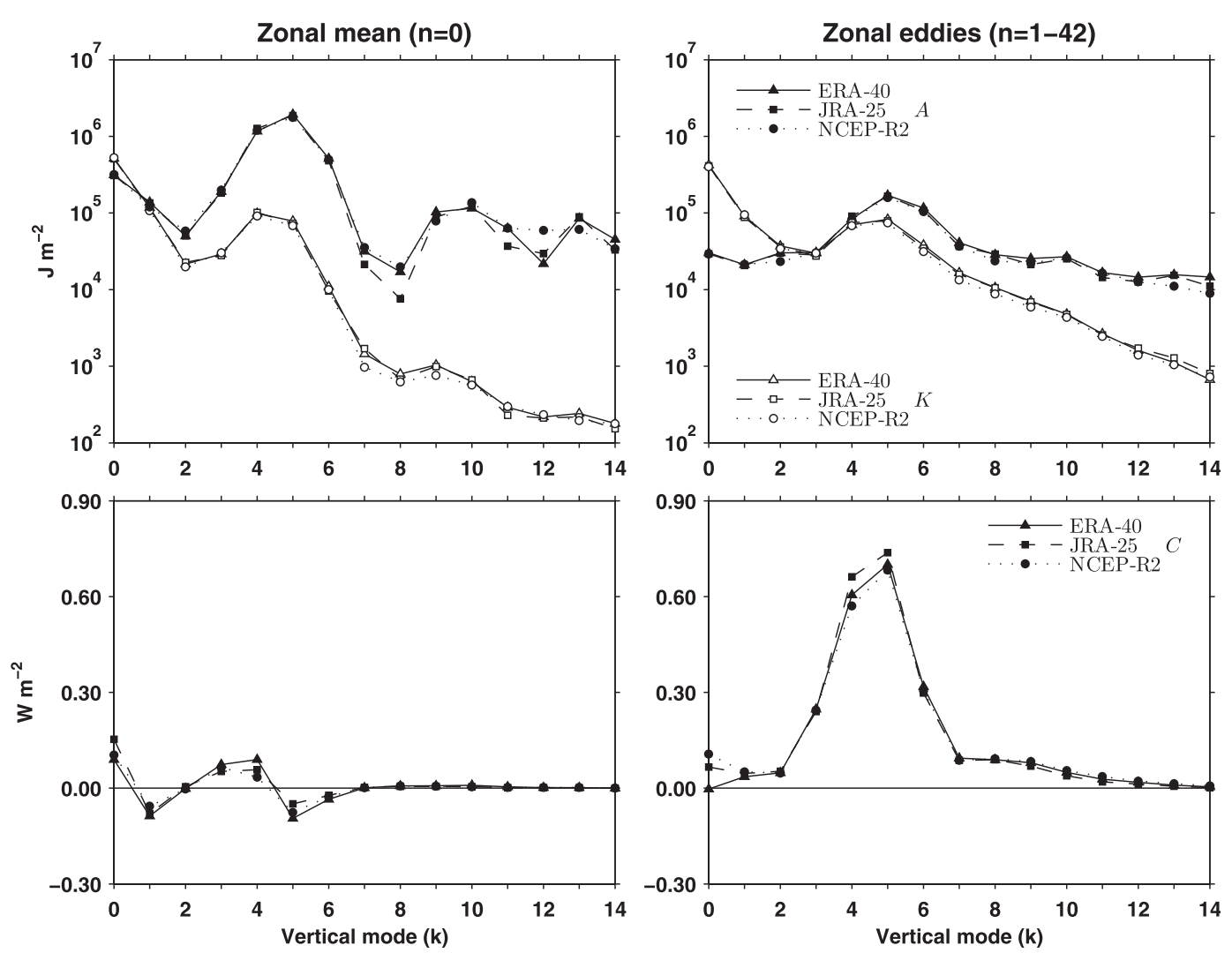

FIG. 2. Spectra of (top) available potential and kinetic energies ( $\left.\mathrm{J} \mathrm{m}^{-2}\right), A$ (black symbols) and $K$ (white symbols), and of (bottom) the conversion rate of available potential energy into kinetic energy $C\left(\mathrm{~W} \mathrm{~m}^{-2}\right)$ in the vertical mode domain for ERA-40, JRA-25, and NCEP-R2 in DJF. The spectra are presented separately for the (left) zonal mean and (right) eddy components.

(white symbols), and (bottom) the spectra for the conversion rate of available potential energy into kinetic energy $C$ in the zonal wavenumber and vertical mode domains, respectively, for ERA-40, JRA-25, and NCEP$\mathrm{R} 2$ in DJF. The contributions from the barotropic and baroclinic vertical modes are presented separately in the left and right columns of Fig. 1, whereas the contributions from the zonal mean and eddy components are illustrated in the left and right columns of Fig. 2, respectively.

The three reanalysis show identical spectra for $A$ and $K$ in the zonal wavenumber domain, except at wavenumbers $n \geqslant 25$ for NCEP-R2. There is a rapid decrease in the spectra of NCEP-R2 at $n \geqslant 36$, previously reported by Watarai and Tanaka (2007) for NCEP-R1 and also by Marques et al. (2010) for NCEP-R2. In addition, it may be seen that both energy spectra for NCEP-R2 starts to decrease more rapidly than those for ERA-40 and JRA-25 at wavenumbers $n \geqslant 25$. Most of $A$ resides in the baroclinic modes, whereas for $K$ there are comparable amounts of energy among the barotropic and baroclinic modes.

Both energy spectra follows approximately the -3 power law for the baroclinic modes in the range of $7 \lessgtr n \leqslant 30$, which is in line with several results reported in the literature (e.g., Charney 1971; Nastrom and Gage 1985; Tanaka 1985; Tanaka et al. 1986; Terasaki and Tanaka 2007a) and has been regarded as an inertial subrange for two-dimensional isotropic turbulence in the atmosphere. For the barotropic mode, the spectra of $K$ follows the -4 power law, which agrees with Terasaki and Tanaka (2007b) for total energy, and can be explained with the Rossby wave saturation theory according to Tanaka et al. (2004), but the slope for the $A$ spectra is steeper, following approximately a -5 power law. This -5 powerlaw behavior in the spectra of $A$ has been observed in the work of Steinberg (1971). Additionally, Merilees and Warn (1972) argue that any numerical model that has a much finer resolution in the horizontal than the vertical would ultimately have a section of the spectrum where available potential energy would follow the -5 power law.

The distribution of available potential energy shows a maximum at $k=5$ for both the zonal mean and eddy components, with the eddy maximum an order of magnitude smaller than that of the zonal mean component. 

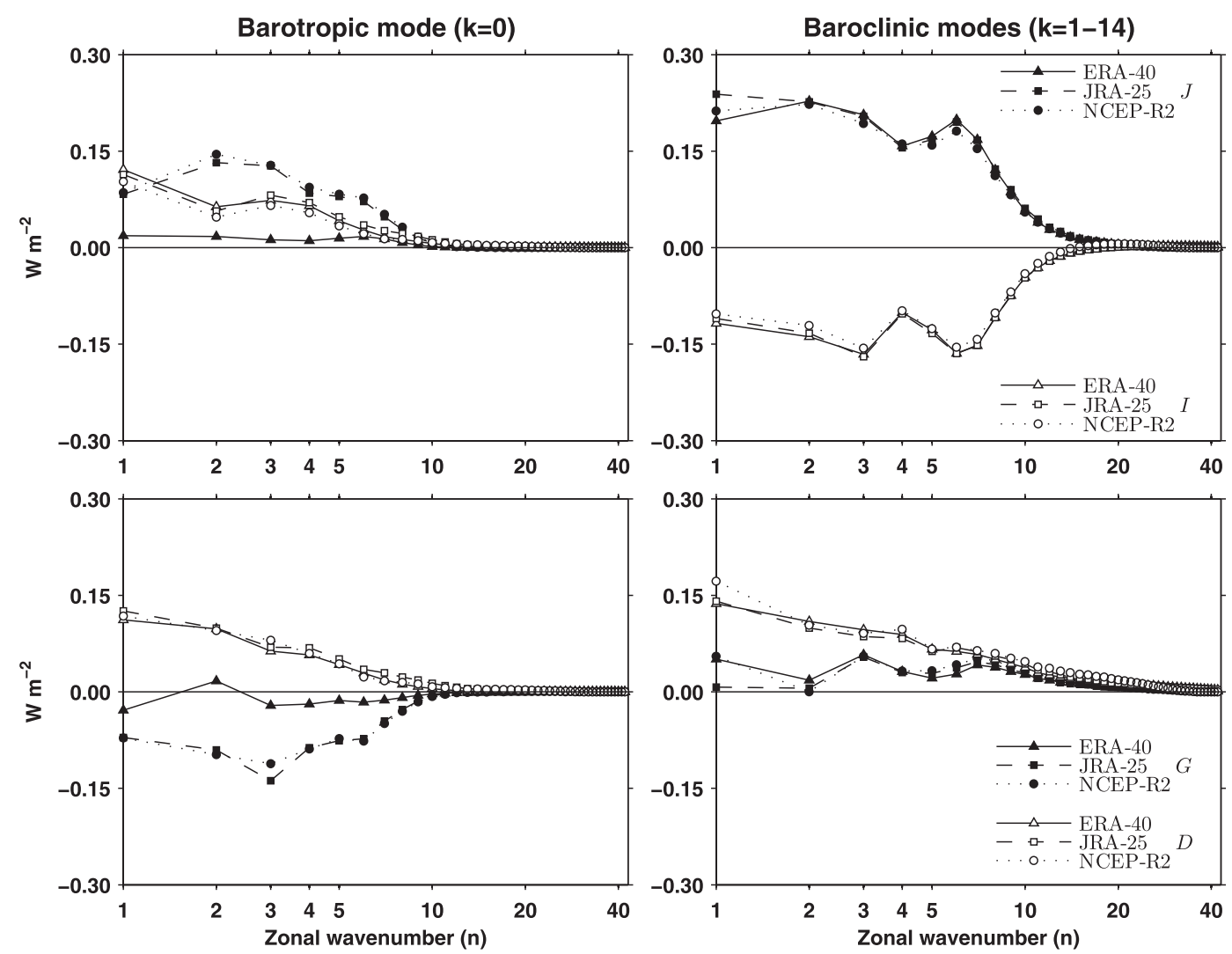

FIG. 3. Zonal wavenumber spectra of (top) interactions of available potential and kinetic energies, $J$ (black symbols) and $I$ (white symbols), and of (bottom) generation and dissipation rates of available potential energy and kinetic energy, $G$ (black symbols) and $D$ (white symbols), respectively. Separation into barotropic and baroclinic modes is as in Fig. 1. Units are $\mathrm{W} \mathrm{m}^{-2}$.

In the zonal mean, there is another peak of energy at $k=$ 0 , while for the eddy component the energy level is small at the barotropic mode. A large amount of kinetic energy is included at the barotropic mode for both the zonal mean and eddy components, with comparable magnitudes. In both components there is another energy peak at $k=4-5$.

The zonal wavenumber spectra of $C$ show that the contributions from the barotropic mode are quite small, and therefore negligible, which confirms the baroclinic nature of this conversion rate. For the baroclinic modes, the spectra of $C$ have maxima at the planetary-scale wavenumbers $n=1-3$, and at the synoptic-scale wavenumber $n=6$, in agreement with several previous studies (e.g., Kung and Tanaka 1983; Hasegawa et al. 1997; Watarai and Tanaka 2007; Marques et al. 2010).

The conversion rate between the zonal mean components of available potential and kinetic energies is relatively small for all reanalyses. It seems that zonal mean available potential energy is converted into zonal mean kinetic energy at the barotropic mode $k=0$ and at the baroclinic modes $k=3-4$, whereas at the baroclinic modes $k=1$ and 5-6 the conversion is in the opposite direction. The spectra for the conversion rate between the eddy components of $A$ and $K$ are in the bottom-right panel of Fig. 2. Despite the nonzero values at $k=0$ (especially for JRA-25 and NCEP-R2), it is seen that this conversion is essentially accomplished by the baroclinic modes, mainly at $k=3-6$ with maximum at $k=5$.

Figures 3 and 4 show (top) the spectra for the interactions of available potential and kinetic energies, $J$ (black symbols) and $I$ (white symbols), along with (bottom) the spectra for the generation and dissipation rates of $A$ and $K, G$ (black symbols) and $D$ (white symbols). As in Figs. 1 and 2, separated components of barotropic and baroclinic modes are shown in Fig. 3, whereas zonal mean and eddy components are shown separately in Fig. 4.

The summations of $J$ and $I$ over all indices $n$ and $k$ should ideally be zero, but these conditions may not be met because of the extrapolation of data below the ground surface, in the reanalysis data, and to numeric and truncation errors. The summations of $J$ yielded imbalances of 

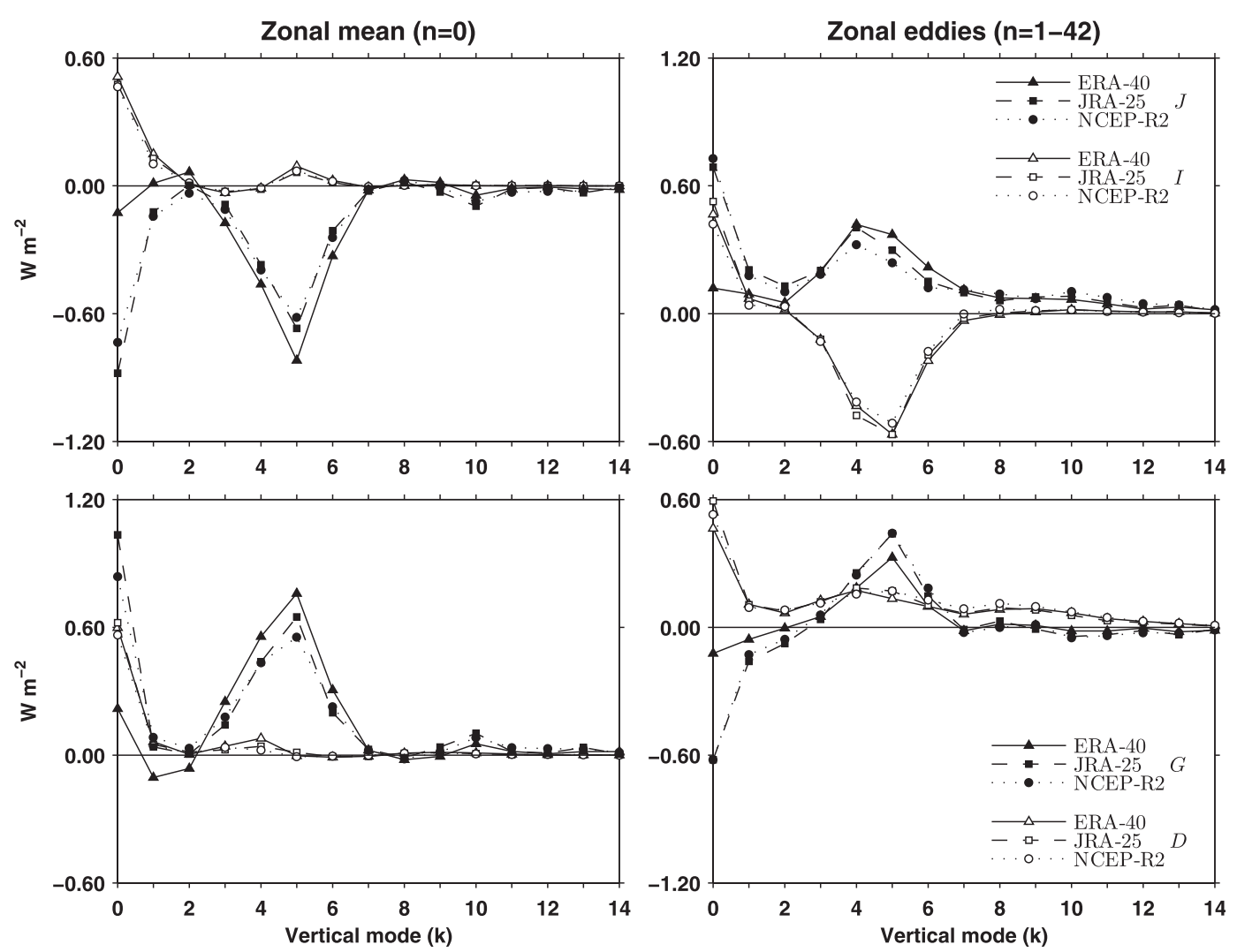

FIG. 4. Spectra of interactions of (top) available potential and kinetic energies, $J$ (black symbols) and $I$ (white symbols), and of (bottom) the generation and dissipation rates of available potential energy and kinetic energy, $G$ (black symbols) and $D$ (white symbols), respectively, in the vertical mode domain. Separation into zonal mean and eddy components is as in Fig. 2.

about $3 \%, 10 \%$, and $8 \%$ for ERA-40, JRA-25, and NCEP-R2, respectively. We thus have adjusted the error by redistributing it over all vertical and zonal wavenumbers, proportionally to their energy interaction. The same approach was adopted for term $I$ because of the imbalances of about $2 \%, 4 \%$, and $3 \%$ for ERA-40, JRA-25, and NCEP-R2, respectively. Since the amount of energy interaction redistributed over each vertical and zonal index is very small, the spectra for the interactions $J$ (or $I$ ) using the adjusted values is nearly identical to the same spectra using the unadjusted values.

The positive values of $J$ in Fig. 3 mean that the zonal mean available potential energy is transferred into the eddy available potential energy, with substantially larger contributions from the baroclinic modes compared with the barotropic mode. In the barotropic mode of ERA-40 this interaction is considerably smaller than those of both JRA-25 and NCEP-R2. Part of the discrepancy may be related to the treatment of data at the lower levels, namely in those regions where the pressure levels are pierced by topography. In the wavenumber domain, $I$ shows negative values for the baroclinic modes, which means that the eddy kinetic energy is transferred out of those modes. On the other hand, there is an input of eddy kinetic energy in the barotropic mode, as seen by the positive values of $I$.

The vertical spectra show negative values of $J$ at both the barotropic $(k=0)$ and baroclinic modes (peaking at $k=5$ ) of the zonal mean component, whereas for the eddy component both the barotropic and baroclinic modes (peaking at $k=4$ ) have positive values. Therefore, the zonal mean available potential energy is transferred into eddy available potential energy both the barotropic and baroclinic. The barotropic interaction in ERA-40 is also considerably smaller than those of JRA-25 and NCEP-R2 at the zonal mean component.

The vertical spectra for $I$ shows that the eddy kinetic energy contained in the baroclinic modes is transferred into the barotropic modes of both zonal mean and eddy components, which agrees with previous studies (e.g., Tanaka 1985; Tanaka and Kung 1988; Terasaki and Tanaka 2007a). A small amount of baroclinic eddy kinetic energy is also transferred into baroclinic zonal mean kinetic energy. 

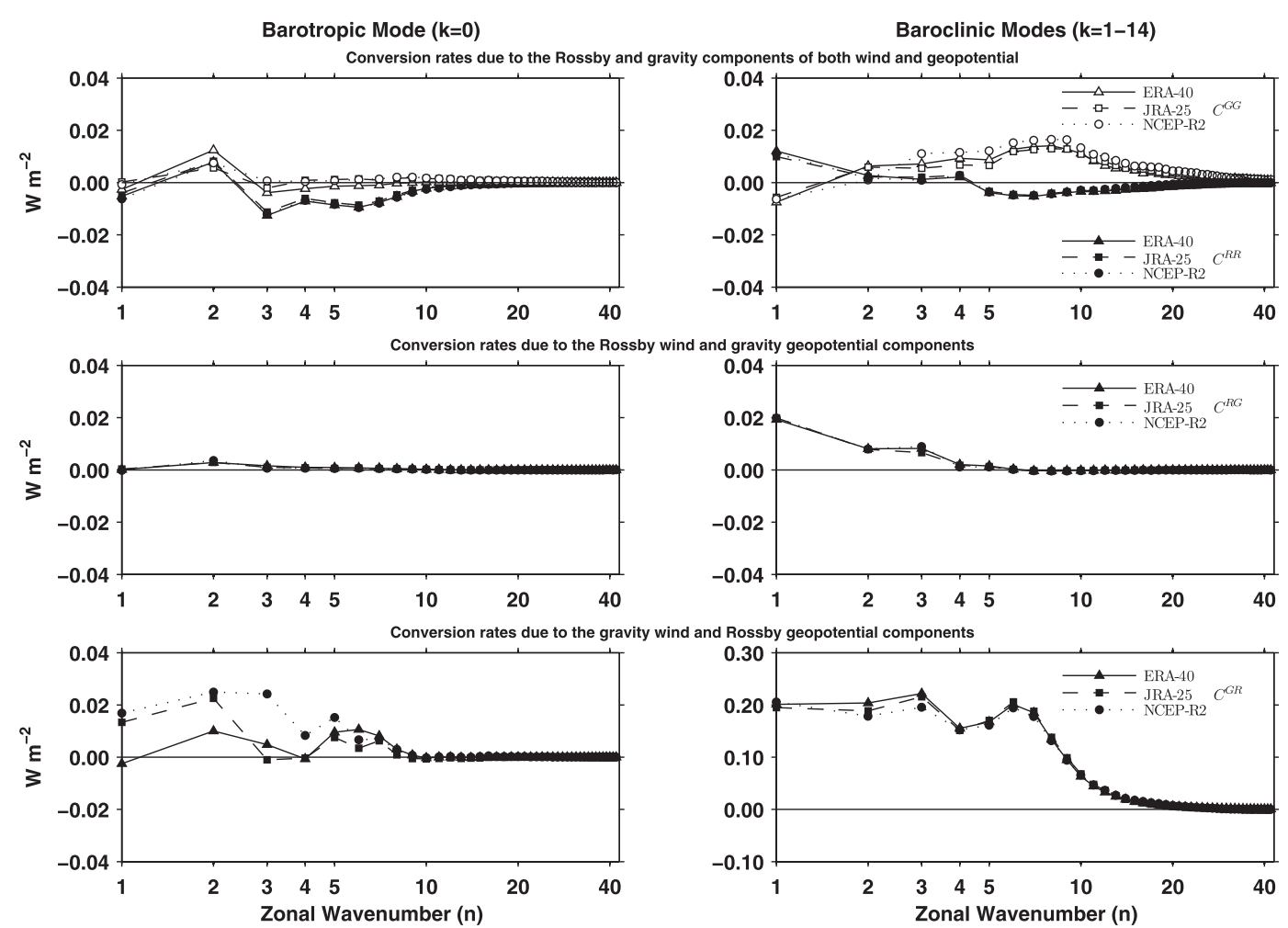

FIG. 5. Zonal wavenumber spectra for the conversion rate of $A$ into $K$ for ERA-40, JRA-25, and NCEP-R2 in DJF. Separation into barotropic and baroclinic modes is as in Fig. 1. The conversion rate is decomposed into terms $C^{R R}$ (black symbols) and $C^{G G}$ (white symbols), respectively due to the Rossby and gravity components of (top) $u, v$, and $\phi$, (middle) term $C^{R G}$, which is due to the Rossby components of $u$, $v$, and the gravity components of $\phi$, and (bottom) term $C^{G R}$ due to the gravity components of $u, v$, and the Rossby components of $\phi$. Units are $\mathrm{W} \mathrm{m}^{-2}$.

Terms $G$ and $D$ were computed as residuals from the balance equations (30) and (35), accounting for the time change of both $A$ and $K$. The results show that the zonal mean available potential energy is generated in the barotropic and baroclinic modes $(k=3-6)$, whereas the eddy available potential energy is generated in the baroclinic modes $(k=4-6)$, with maximums at wavenumbers $n=3$ and 7 , but it is dissipated $(G<0)$ in the barotropic mode by the planetary and synopticscale eddies $(n \simeq 1-10)$. Consistently with interactions $J$, the absolute values of energy generation in both the zonal mean and eddy barotropic modes of ERA-40 are substantially smaller than those of JRA-25 and NCEP-R2. The eddy available potential energy dissipated at $k=0$ is approximately of the same amount as the energy supplied from the zonal mean component into the barotropic mode of the eddy component. The eddy kinetic energy is mostly dissipated by the planetary- and synopticscale eddies in the barotropic and baroclinic modes, whereas the zonal mean kinetic energy is dissipated mainly in the barotropic mode, with only a small amount dissipated in the baroclinic modes.

\section{b. Decomposition of the conversion rates into contributions from Rossby and gravity fields}

The conversion rates of available potential energy into kinetic energy were decomposed into the contributions from the Rossby and gravity fields using Eq. (43), with detailed expressions for its terms given in Eqs. (44)-(47). The results of such a decomposition are presented in Figs. 5 and 6 . The conversion rates due to the Rossby components of the wind field $(u, v)$ and geopotential $\phi$, denoted as $C^{R R}$, and those due to the gravity components of $u, v$, and $\phi$, denoted as $C^{G G}$, are represented in the top panels, whereas the conversion rates due to the Rossby components of $u$ and $v$ and the gravity components of $\phi$ (i.e., $C^{R G}$ ) and those due to the gravity components of $u$ and $v$ and the Rossby components of $\phi$ (i.e., $C^{G R}$ ) are in the middle and bottom panels, respectively. Comparing the magnitudes of the different terms, it may be seen that the conversion of available potential energy into kinetic energy is essentially accomplished by the work realized on the divergent motion-that is, by the terms involving the gravity wind, namely the 

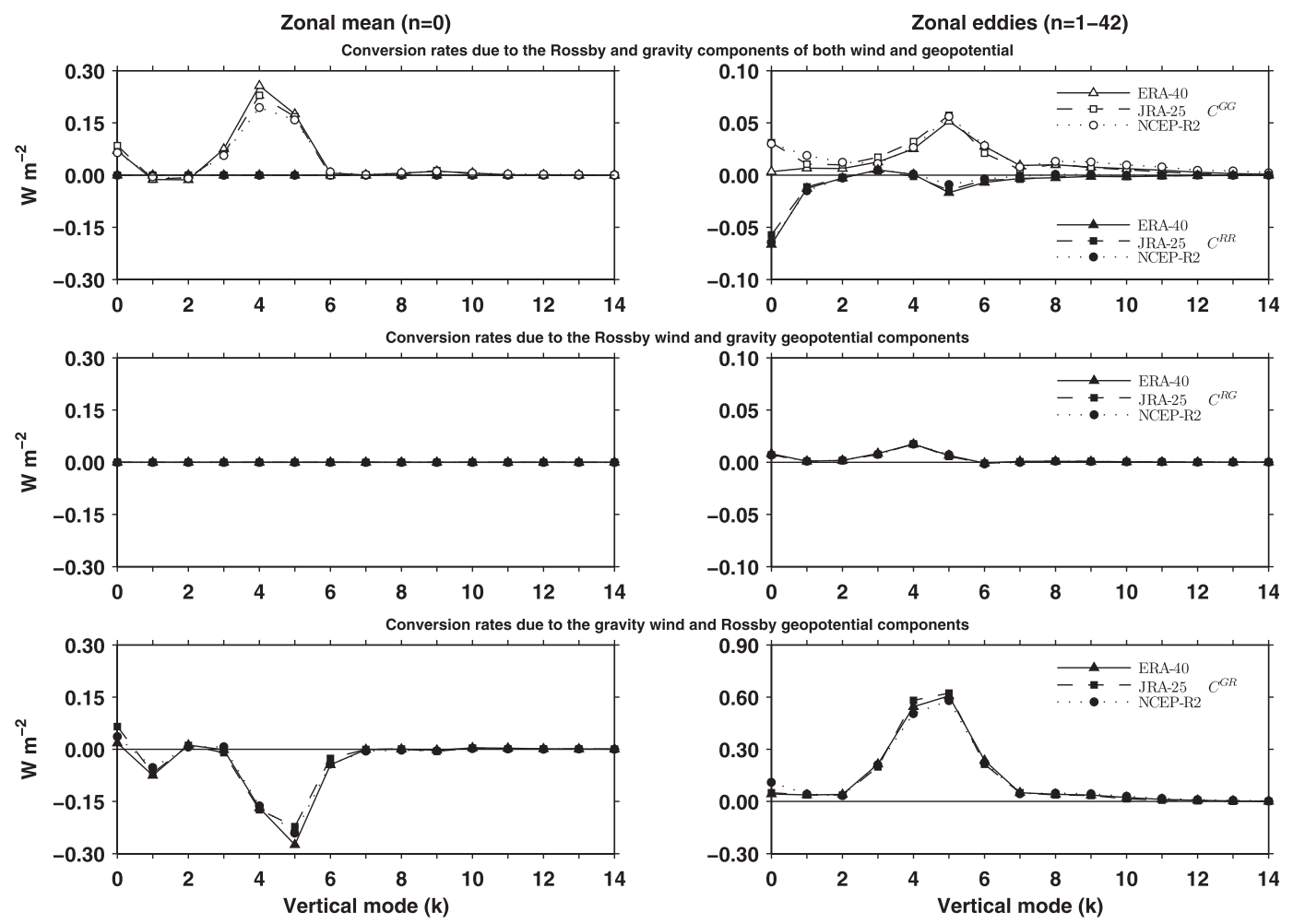

FIG. 6. As in Fig. 5, but for the spectra in the vertical mode domain. Separation into zonal mean and eddy components is as in Fig. 2.

zonal mean component of $C^{G G}$ and the zonal mean and eddy components of $C^{G R}$. Because the zonal mean terms nearly cancel each other out, the net conversion rate is mostly due to the eddy component of term $C^{G R}$. In other words, the net conversion rate of available potential energy into kinetic energy is mostly due to the conversion of potential energy of the rotational adjusted mass field into kinetic energy by the work realized in the eddy divergent motion. The nearly opposite conversions of zonal mean components of $C^{G G}$ and $C^{G R}$ may be interpreted as a geostrophic adjustment with the potential energy of divergent field being converted into potential energy of zonal mean geostrophic (rotational) field. The zonal mean components of terms $C^{R R}$ and $C^{R G}$ are zero, as expected.

\section{ENERGY CYCLE DIAGRAM IN THE ZONAL WAVENUMBER AND VERTICAL MODE DOMAINS}

Using the results presented in the previous subsections, it is possible to construct a detailed energy cycle diagram describing the flow of energy among the zonal mean and eddy components, and also among the barotropic and baroclinic components. That is, the available potential and kinetic energies are decomposed into the zonal mean $(n=0)$ and eddy $(n \geq 1)$ components, denoted respectively by subscripts $Z$ and $E$ (i.e., $A_{Z}, A_{E}, K_{Z}$, and
$\left.K_{E}\right)$ and each of these components is also decomposed into the barotropic $(k=0)$ and baroclinic $(k \geq 1)$ components, which are denoted by the extra subscripts $B$ and $b$, respectively. Thus, for example, the term $A_{Z B}$ denotes the zonal mean available potential energy of the barotropic component. The terms representing the energy conversion rates and the generation or dissipation rates are denoted similarly. For example, the term $C_{Z B}$ denotes the conversion rate between the energies contained in the zonal barotropic components, which are $A_{Z B}$ and $K_{Z B}$, whereas $G_{Z B}\left(D_{Z B}\right)$ is the generation (dissipation) rate of available potential (kinetic) energy in the zonal mean barotropic component. The zonal-wave interactions of available potential and kinetic energies are represented by symbols $J$ and $I$, respectively. The barotropic-barotropic interactions are denoted with subscript $B$ (i.e., $J_{B}$ and $I_{B}$ ) whereas the baroclinicbaroclinic interactions are denoted with subscript $b$ (i.e., $J_{b}$ and $I_{b}$ ). For the barotropic-baroclinic interactions the symbols $J$ and $I$ are used with the origin and destination of energy specified by an appropriate combination of letters $Z, E, B$, and $b$ between parentheses. For example, $J(Z B$, $E b)$ designates the transfer rate of available potential energy from the zonal mean barotropic component $A_{Z B}$ to the eddy baroclinic component $A_{E b}$. 


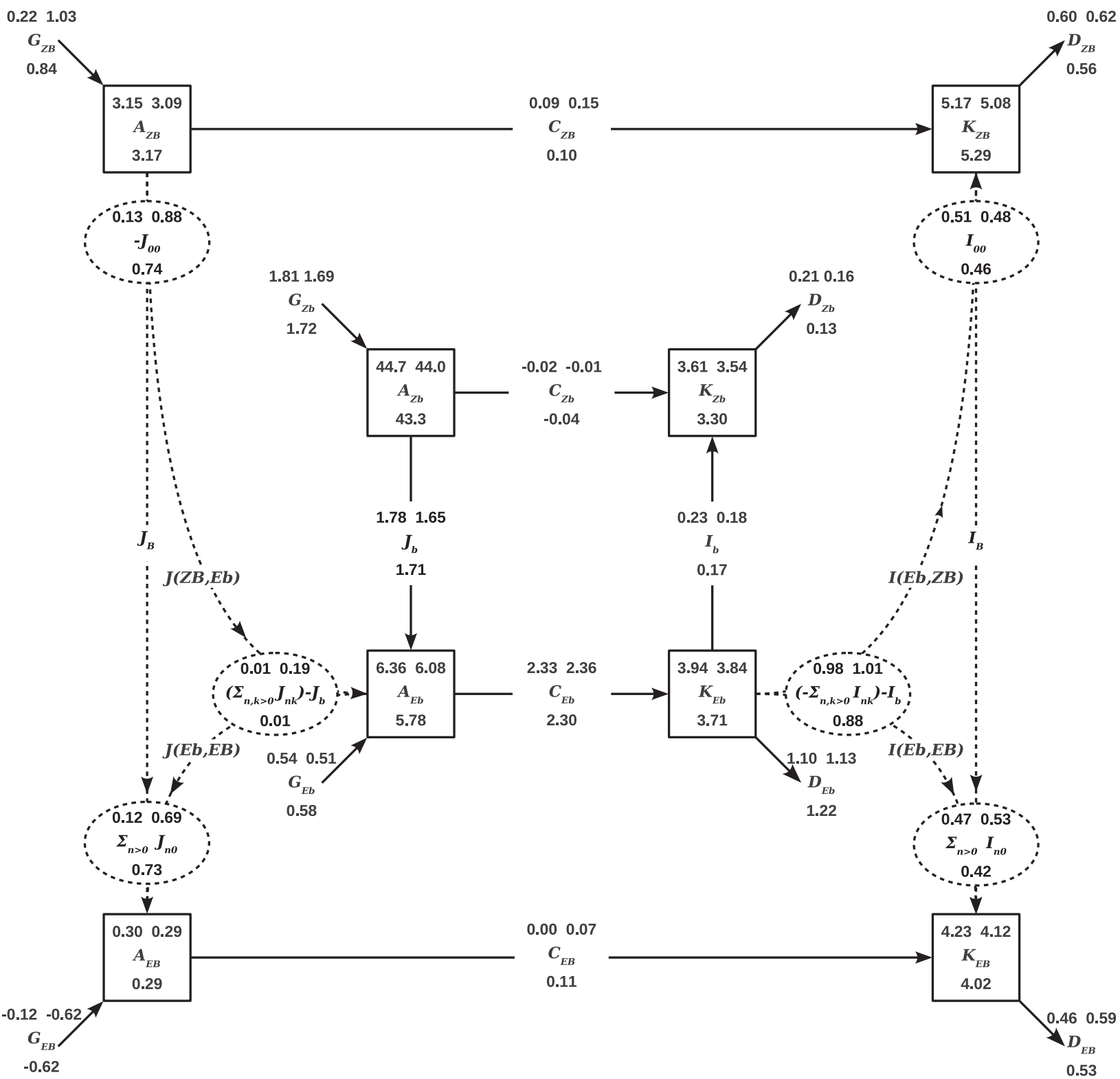

FIG. 7. Extended energy cycle diagram describing the flow of energy among the zonal mean and eddy components, and also among the barotropic and baroclinic components for ERA-40 (top-left values), JRA-25 (top-right values), and NCEP-R2 (bottom values) in DJF climate. Units are $10^{5} \mathrm{~J} \mathrm{~m}^{-2}$ for energy levels and $\mathrm{W} \mathrm{m}^{-2}$ for conversion/transfer rates and generation/dissipation rates.

Such a detailed energy cycle diagram is illustrated in Fig. 7, in which the boxes represent the levels of energy and the arrows the energy generation/dissipation rates and the energy conversion/transfer rates. The estimates in the diagram are for the ERA-40 (top-left value), JRA-25 (top-right value), and NCEP-R2 (bottom value) in the DJF climate. A negative value means that the energy flows in the opposite direction of that indicated by the arrow. The expressions for all the terms in Fig. 7, which are obtained from the formulas given in section 2 , are displayed in the appendix.
The inner four boxes in Fig. 7 represent the energy of baroclinic reservoirs. The nonlinear energy interactions, and the energy conversions between them, show a picture of the energy cycle similar to the traditional Lorenz cycle with available potential energy generated in the zonal flow. This energy is transferred to the baroclinic eddies by nonlinear interactions and converted into kinetic energy. Part of the kinetic energy is dissipated in the baroclinic eddies and the other part is transferred to the zonal mean baroclinic circulation where it is dissipated. The similarity is only in the sense of the energy flow; the magnitude of 
the terms is different. The partition of the energy reservoirs into barotropic and baroclinic components allows for more detail in the processes involved. It is observed that there is generation of available potential energy in the baroclinic and also in the zonal mean barotropic component. Part of the available potential energy is dissipated in the barotropic eddies, probably by the warming of cold air masses and the cooling of warm air masses in middle latitudes (Lorenz 1955). The large fraction of the generated available potential energy is converted to kinetic energy in the baroclinic eddies. Almost half of the converted kinetic energy is dissipated by surface friction and viscosity in the baroclinic eddies. The remaining kinetic energy is almost entitely transferred by nonlinear interactions to the barotropic components, except a small fraction that is transferred into the zonal mean baroclinic component. This energy is ultimately dissipated, in the three reservoirs, by surface friction and viscosity. [At first view it may seems strange that there is generation of available potential energy in the zonal mean barotropic component. However, one must be aware that when barotropy is mentioned, it is, in fact, an equivalent barotropy with the atmosphere thicker (hotter) in the tropics and thinner (colder) at high latitudes but with the meridional gradient of temperature not strong enough to cause baroclinic instability (e.g., Cehelsky and Tung 1991).]

In the normal-mode scheme presented here, the computation of the nonlinear interactions only allows us to identify the inputs of energy in each reservoir, it being impossible to identify the reservoir from which the energy flows. Therefore, it is only possible to know the energy balance of different processes. The values inside the dotted ellipses represent the balances of the flows of kinetic energy and available potential energy between the zonal barotropic component and the barotropic and baroclinic eddies. The energy flows represented by the dotted lines cannot by quantified individually. These flows are associated with eddy generation by barotropic instability (e.g., Jacobs and Wiin-Nielsen 1966) and the barotropic decay of baroclinic eddies (e.g., Tanaka 1995; Mak 2000).

\section{Concluding remarks}

An energetics formulation has been introduced that enables an explicit evaluation for the conversion rates between available potential energy and kinetic energy, the nonlinear interactions of both energy forms, and their generation and dissipation rates, in both the zonal wavenumber and vertical mode domains. In addition, we presented a decomposition for the conversion rates of available potential energy into kinetic energy, according to the contributions of the Rossby and gravity components of geopotential and horizontal wind fields. Finally, we also proposed an extended energy cycle diagram describing the flow of energy among the zonal mean and eddy components, and also among the barotropic and baroclinic components. Using these schemes, an energetics analysis was assessed for the DJF climate from three reanalysis datasets (ERA-40, JRA-25, and NCEP-R2).

The detailed energy scheme proposed here allows us to assess characteristics of the energy cycle not accessible using other schemes found in the literature. For example, it was seen that the energy supplied into the barotropic mode of the eddy component is almost entirely dissipated there. The conversion rate of available potential energy into kinetic energy is essentially due to the baroclinic conversion of potential energy of the rotational adjusted mass field into kinetic energy by the work realized in the eddy divergent motion. The near absence of a net conversion rate between the zonal mean components of available potential and kinetic energies is the consequence of a geostrophic adjustment, with the available potential energy in the divergent mass field being converted into kinetic energy of the divergent zonal mean meridional motion, which in turn is converted back into available potential energy of the zonal mean geostrophic mass field.

On the whole, the energetics analysis assessed for the DJF climate is consistent among the three reanalysis datasets. The peaks and slopes in the spectra of the various components and the inputs and outputs of energy generally agree among the three reanalyses. Nevertheless, some differences were also found among the three reanalyses, in both the qualitative and quantitative senses, which worth mentioning. There is a rapid decrease in the wavenumber energy spectra of NCEP-R2 at $n \geq 36$ for both barotropic and baroclinic modes. These spectra start to decrease more rapidly for NCEP-R2 than those for ERA-40 and JRA-25 even at wavenumbers $n \geq 25$. The values for the nonlinear interactions of available potential energy in ERA-40 are substantially smaller than those in JRA-25 and NCEP-R2 for the barotropic modes of both the zonal mean and eddy components. Since the barotropic component of $J$ seems to be sensitive to the treatment of the lower boundary, part of this discrepancy may be related to the use of different methods, among the reanalysis systems, for data interpolation/extrapolation from model levels into pressure levels at the lower atmosphere. From the residual estimates, the net generation among the barotropic and baroclinic components yields an input of eddy available potential energy in ERA-40, whereas in JRA-25 and NCEP-R2 the same estimates give a small output of eddy available potential energy.

The analysis schemes presented in this study can therefore complement the traditional wavenumber energetics of Saltzman (1957) and the 3D NME introduced by Tanaka (1985), and may be a useful tool for future 
diagnosis of reanalysis data and to assess the performance of climate models with additional detail from the energetics point of view. The differences between the three reanalyses used here seem to be reasonably unremarkable, and since similar data go into each of the reanalysis products, some discrepancies found between them should be mostly attributed to the different model resolutions and model biases and the different data assimilation methods among them. Finally, we note that this scheme can be applied using sigma coordinates with the available potential energy defined as in Kasahara and Puri (1981), which has the advantage of avoiding the problems associated with topography in isobaric coordinates.

Acknowledgments. C. A. F. Marques was supported by the Portuguese Foundation for Science and Technology (FCT) under Grant SFRH/BPD/76232/2011. The ERA-40 data were obtained from the ECMWF data server. The JRA-25 datasets used for this study were provided from the cooperative research project of the JRA-25 long-term reanalysis by the Japan Meteorological Agency (JMA) and the Central Research Institute of Electric Power Industry (CRIEPI). NCEP/DOE-R2 data provided by the NOAA/OAR/ESRL PSD, Boulder, Colorado, USA, were extracted from their website (at http://www.cdc.noaa.gov/).

\section{APPENDIX}

\section{List of Symbols in the Extended Energy Cycle Diagram}

The terms in Fig. 7 are obtained with the following expressions:

$$
\begin{aligned}
& A_{Z B}=A_{00}, \\
& A_{Z b}=\sum_{k=1}^{\mathcal{K}} A_{0 k}, \\
& A_{E B}=\sum_{n=1}^{\mathcal{N}} A_{n 0}, \\
& A_{E b}=\sum_{n=1}^{\mathcal{N}} \sum_{k=1}^{\mathcal{K}} A_{n k}, \\
& K_{Z B}=K_{00}, \\
& K_{Z b}=\sum_{k=1}^{\mathcal{K}} K_{0 k}, \\
& K_{E B}=\sum_{n=1}^{\mathcal{N}} K_{n 0},
\end{aligned}
$$

$$
\begin{aligned}
& K_{E b}=\sum_{n=1}^{\mathcal{N}} \sum_{k=1}^{\mathcal{K}} K_{n k} \\
& C_{Z B}=C_{00} \text {, } \\
& C_{Z b}=\sum_{k=1}^{\mathcal{K}} C_{0 k} \\
& C_{E B}=\sum_{n=1}^{\mathcal{N}} C_{n 0} \\
& C_{E b}=\sum_{n=1}^{\mathcal{N}} \sum_{k=1}^{\mathcal{K}} C_{n k} \\
& J_{B}+J(Z B, E b)=-J_{00}, \\
& J_{B}+J(E b, E B)=\sum_{n=1}^{\mathcal{N}} J_{n 0}, \\
& J(Z B, E b)-J(E b, E B)=\sum_{n=1}^{\mathcal{N}} \sum_{k=1}^{\mathcal{K}} J_{n k}+\sum_{k=1}^{\mathcal{K}} J_{0 k}, \\
& J_{b}=-\sum_{k=1}^{\mathcal{K}} J_{0 k} \\
& I_{b}=\sum_{k=1}^{\mathcal{K}} I_{0 k} \\
& I(E b, Z B)+I(E b, E B)=-\sum_{n=1}^{\mathcal{N}} \sum_{k=1}^{\mathcal{K}} I_{n k}-\sum_{k=1}^{\mathcal{K}} I_{0 k}, \\
& I(E b, Z B)-I_{B}=I_{00} \\
& I(E b, E B)+I_{B}=\sum_{n=1}^{\mathcal{N}} I_{n 0} \\
& G_{Z B}=-J_{00}+C_{00}+\frac{\partial}{\partial t} A_{00}, \\
& G_{Z b}=\sum_{k=1}^{\mathcal{K}}\left(-J_{0 k}+C_{0 k}+\frac{\partial}{\partial t} A_{0 k}\right), \\
& G_{E B}=\sum_{n=1}^{\mathcal{N}}\left(-J_{n 0}+C_{n 0}+\frac{\partial}{\partial t} A_{n 0}\right), \\
& G_{E b}=\sum_{n=1}^{\mathcal{N}} \sum_{k=1}^{\mathcal{K}}\left(-J_{n k}+C_{n k}+\frac{\partial}{\partial t} A_{n k}\right),
\end{aligned}
$$




$$
\begin{aligned}
& D_{Z B}=I_{00}+C_{00}-\frac{\partial}{\partial t} K_{00}, \\
& D_{Z b}=\sum_{k=1}^{\mathcal{K}}\left(I_{0 k}+C_{0 k}-\frac{\partial}{\partial t} K_{0 k}\right), \\
& D_{E B}=\sum_{n=1}^{\mathcal{N}}\left(I_{n 0}+C_{n 0}-\frac{\partial}{\partial t} K_{n 0}\right), \text { and } \\
& D_{E b}=\sum_{n=1}^{\mathcal{N}} \sum_{k=1}^{\mathcal{K}}\left(I_{n k}+C_{n k}-\frac{\partial}{\partial t} K_{n k}\right),
\end{aligned}
$$

where the vertical mode and zonal wavenumber have been truncated at $\mathcal{K}=14$ and $\mathcal{N}=42$, respectively.

\section{REFERENCES}

Castanheira, J. M., C. C. DaCamara, and A. Rocha, 1999: Numerical solutions of the vertical structure equation and associated energetics. Tellus, 51A, 337-348.

Cehelsky, P., and K. K. Tung, 1991: Nonlinear baroclinic adjustment. J. Atmos. Sci., 48, 1930-1947.

Charney, J. G., 1971: Geostrophic turbulence. J. Atmos. Sci., 28, $1087-1095$.

Cohn, S. E., and D. P. Dee, 1989: An analysis of the vertical structure equation for arbitrary thermal profiles. Quart. J. Roy. Meteor. Soc., 115, 143-171.

Daley, R., 1991: Atmospheric Data Analysis. Cambridge University Press, $457 \mathrm{pp}$.

Eliasen, E., and B. Machenhauer, 1965: A study of the fluctuations of the atmospheric planetary flow patterns represented by spherical harmonics. Tellus, 17, 220-238.

Hasegawa, A., H. L. Tanaka, H. Hirakuchi, and S. Taguchi, 1997: Comparative energetics analysis of CCM2 with different horizontal resolutions. Climate Dyn., 13, 521-532.

Hayashi, Y., 1980: Estimation of nonlinear energy transfer spectra by the cross-spectral method. J. Atmos. Sci., 37, 299-307.

Holmström, I., 1963: On a method for parametric representation of the state of the atmosphere. Tellus, 15, 127-149.

Jacobs, S. J., and A. Wiin-Nielsen, 1966: On the stability of a barotropic basic flow in a stratified atmosphere. J. Atmos. Sci., 23, 682-687.

Kao, S. K., 1968: Governing equations and spectra for atmospheric motion and transports in frequency-wavenumber space. J. Atmos. Sci., 25, 32-38.

Kasahara, A., and K. Puri, 1981: Spectral representation of threedimensional global data by expansion in normal mode functions. Mon. Wea. Rev., 109, 37-51.

Kung, E. C., and H. Tanaka, 1983: Energetics analysis of the global circulation during the special observation periods of FGGE. J. Atmos. Sci., 40, 2575-2592.
Lambert, S. J., 1984: A global available potential energy-kinetic energy budget in terms of the two-dimensional wavenumber for the FGGE year. Atmos.-Ocean, 22, 265-282.

Lorenz, E. N., 1955: Available potential energy and the maintenance of the general circulation. Tellus, 7, 157-167.

Mak, M., 2000: Does an unstable baroclinic wave equilibrate/decay baroclinically or barotropically? J. Atmos. Sci., 57, 453-463.

Marques, C. A. F., A. Rocha, and J. Corte-Real, 2010: Comparative energetics of ERA-40, JRA-25 and NCEP-R2 reanalysis, in the wave number domain. Dyn. Atmos. Oceans, 50, 375-399.

Merilees, P. E., and T. Warn, 1972: The resolution implications of geostrophic turbulence. J. Atmos. Sci., 29, 990-991.

Nastrom, G. D., and K. S. Gage, 1985: A climatology of atmospheric wavenumber spectra of wind and temperature observed by commercial aircraft. J. Atmos. Sci., 42, 950-960.

Saltzman, B., 1957: Equations governing the energetics of the larger scales of atmospheric turbulence in the domain of wave number. J. Meteor., 14, 513-523.

Shigehisa, Y., 1983: Normal modes of the shallow water equations for zonal wavenumber zero. J. Meteor. Soc. Japan, 61, 479-494.

Steinberg, H. L., 1971: On power laws and nonlinear cascades in large scale atmospheric flow. Tech. Rep. 002630-4-T, Dept. of Meteorology and Oceanography, University of Michigan, $162 \mathrm{pp}$.

Swarztrauber, P. N., and A. Kasahara, 1985: The vector harmonic analysis of Laplace's tidal equations. SIAM J. Sci. Stat. Comput., 6, 464-491.

Tanaka, H. L., 1985: Global energetics analysis by expansion into three-dimensional normal-mode functions during the FGGE winter. J. Meteor. Soc. Japan, 63, 180-200.

_ 1994: Normal mode energetics of the general circulation during the FGGE winter. Sci. Rep. Inst. Geosci. Univ. Tsukuba, 15, 1-19. [Available online at http://air.geo.tsukuba.ac.jp/ tanaka/papers/paper39.pdf.]

, 1995: A life-cycle of nonlinear baroclinic waves represented by a simple 3D spectral model. Tellus, 47A, 697-704.

— circulation during the FGGE year. J. Atmos. Sci., 45, 37233736.

- —, and W. E. Baker, 1986: Energetics analysis of the observed and simulated general circulation using threedimensional normal mode expansions. Tellus, 38A, 412-428.

_- Y. Watarai, and T. Kanda, 2004: Energy spectrum proportional to the squared phase speed of Rossby modes in the general circulation of the atmosphere. Geophys. Res. Lett., 31, L13109, doi:10.1029/2004GL019826.

Terasaki, K., and H. L. Tanaka, 2007a: An analysis of the 3-D atmospheric energy spectra and interactions using analytical vertical structure functions and two reanalyses. J. Meteor. Soc. Japan, 85, 785-796.

$\longrightarrow$, and — 2007b: Barotropic energy spectrum by the Rossby wave saturation in the zonal wavenumber domain. SOLA, $\mathbf{3}$, 25-28.

Watarai, Y., and H. L. Tanaka, 2007: Characteristics of the JRA-25 dataset from the viewpoint of global energetics. SOLA, 3, 9-12. 